\title{
Studying the Chemical Reactivity Properties of Cytosine and its Antiviral Analogues: Zebularine, 5- Aza-Cytosine And 5-Aza-5,6-Dihydro-Cytosine through Density Functional Theory
}

guixiu wang ( $\nabla$ wanggx247@163.com )

Heze University https://orcid.org/0000-0001-7648-9461

Yifang Wu

Heze University

\section{Research Article}

Keywords: cytosine, zebularine, 5-aza-cytosine, 5-aza-5,6-dihydro-cytosine, chemical reactivity

Posted Date: June 21st, 2021

DOl: https://doi.org/10.21203/rs.3.rs-586083/v1

License: (c) (1) This work is licensed under a Creative Commons Attribution 4.0 International License. Read Full License 


\section{Abstract}

Zebularine, 5-aza-cytosine and 5-aza-5,6-dihydro-cytosine are structurally similar to cytosine, but their biological functions are rather different. Cytosine can be methylated which is a gene lesion that can cause human disease. On the contrary, zebularine and 5-aza-cytosine are inhibitors of DNA methylation. 5-aza-5,6-dihydro-cytosine is specifically designed to induce lethal mutagenesis in HIV for its structurally variability. Here, theoretical research into their chemical properties through density functional theory is reported. Molecular hardness and molecular electronic surface potential were analysed. Compared to cytosine, the main reason for the inability of methyl addition of zebularine is the reduced nucleophilicity of $\mathrm{C} 5$ atom. The lack of a hydrogen atom at N5 atom in 5-aza-cytosine is responsible for the incomplete reaction of methyl transfer. Variability of 5-aza-5,6-dihydro-cytosine is responsible for the mutagenesis treatment by paring with guanine or adenine with its different tautomers. Aspect of these chemical reactivities can be accounted for the distinctive biological functions of these molecules.

\section{Introduction}

Nucleoside analogues as agents for anticancer and antiviral therapeutics have been studied and used for fifty years[1]. The naturally occurring nucleosides represent a unique starting point for drug design due to their involvement in numerous critical biological processes as well as the fact that they serve as essential building blocks for DNA/RNA synthesis. On such a point of view, modifications of natural nucleobases can be designed or refined, based on the interactions identified in the binding site of target enzymes. Of the four nitrogenous bases of DNA, cytosine was selected for discussion since an abundance of mutagenesis data concerning this base are available in the scientific literature[2]. Modifications of cytosine would lead to disruption or termination of replication or other biological processes[3]. Here zebularine, in the absence of the amino group at $\mathrm{C} 4$ position, and 5-aza-cytosine analogue (termed as 5azaC, also known as decitabine), modified at $\mathrm{C} 5$ position by an atom $\mathrm{N}$, and its saturated form 5-aza-5,6dihydro-2'-deoxycytosine (5-azaHC) are reported. Cytosine is introduced a methyl group via C5 position catalysed by methyltransferase enzymes[4-6], while the three analogues are the most important nucleoside analogue inhibitors of cytosine methylation[7-9]. The addition of the extra nitrogen also endowed 5-azaC with profound anticancer properties[10-13]. 5-azaHC is known to be incorporated into a viral (HIV) genome to extinguish HIV viruses by elevating its already high mutation rates above the error catastrophe limit, in which limit no viable progeny can be sustained and the viral population collapses[14$16]$.

These different biological functions are closely related to their specific molecular structures. Amino group is an important biologically substituent. The absence of the amino group (zebularine) or nitrogen substitution to the structure of cytosine (5-azaC and 5-azaHC) can have profound effects. The motivation for the present study was to quantify the chemical reactivity parameters of the geometrically similar molecules: zebularine, Cytosine, 5-azaC and 5-azaHC. The properties of these molecules are discussed here emphasizing their different aspects dependent of chemical hardness, and surface electrostatic potential. 


\section{Theoretical Background}

\section{Theoretical background:}

\section{Reactivity parameter}

In density functional theory, the ground-state properties of a many-electron system are uniquely determined by its electron density $\rho(r)$ as expressed below[17]

$$
E[\rho]=F[\rho]+\int \mathcal{U}(r) \rho(r) d r
$$

Where $u(r)$ is the external potential that includes the nuclear potential, and $F[\rho]$ is the universal Hohenberg-Kohn functional composed of the electronic kinetic energy and the electron-electron interaction energy. The global hardness $\eta$ is defined as the second partial derivative of $E[r]$ with respect to the number of electrons $N$ at constant external potential, and an approximation to $\eta$ is used by a finite difference method[18, 19].

$$
\eta=\left(\frac{\partial^{2} E}{\partial N^{2}}\right)_{u(r)} ; \frac{1}{2}(I-E A)
$$

Where $E, I P$ and $E A$ are respectively the total energy, ionization potential and electronic affinity of an $N$ electron system.

\section{Molecular Electrostatic Surface Potential (MESP)}

Electrostatic potential is an important property that plays a key role in the interaction of molecules and is well established as an effective tool for interpreting and predicting molecular reactive behaviour[20-22]. The electronic potential of a molecule is an expression of Coulomb's law:

$$
V_{\text {tot }}(\mathrm{r})=V_{m u}(\mathrm{r})+V_{\text {ele }}(\mathrm{r})=\sum_{A} \frac{Z_{A}}{\left|\mathrm{r}-R_{A}\right|}-\int \frac{\rho\left(\mathrm{r}^{\prime}\right)}{\left|\mathrm{r}^{\prime} \mathrm{r}^{\prime}\right|} d \mathrm{r}^{\prime}
$$

where $Z_{A}$ is the charge on nucleus $A$, located at $R_{A}$. is the electronic density of the molecule and $r^{\prime}$ is a dummy integration variable. Depending on whether the effect of the nuclei or the electrons is dominant in any given region, may be either positive or negative. The most negative-valued points in the MESP topography, usually indicated with the notation $V_{\min }$ is widely used to gauge the electron donating properties of a molecule[23]. Similarly, $\mathrm{V}_{\max }$ is used as a parameter to measure the electron drawing abilities of a molecule[24, 25]. 


\section{Computational Methods}

Hybrid meta-GGA functional M06-2X has been performed to study the different reactivity descriptors concerning IP, electronic affinity, hardness. This method has been proved to give the best correlation with experimental data[26, 27]. The split-valence $6-311++G(2 d, 2 p)$ has been employed in this study. To reduce computational complexity, N1 was substituted by a methyl group instead a nucleoside for these molecules. The geometries of all the molecules were optimized to find the neutral ground electron states. The spin multiplicity corresponding to singlet and doublet has been considered for the ground, cationic and anionic forms of the molecules, respectively. All calculations were performed with Gaussian 09 software[28]. The wave function generated in the computations is used for the calculation of the MESP.

\section{Results And Discussion}

The calculated chemical reactivity parameters for the interested molecules are listed in Table 1. They are ionization potential (IP), defined as the first ionization potential which is used to measure the extraction energy of the outermost electron of an atom of the system and thus indirectly reveal how tightly an electron is bound within the nuclear attractive field; electron affinity (EA), which measures the ability of a molecule to accept an electron and form anions; chemical hardness ( $\eta$ )[29], a descriptor shows the resistance of the chemical potential of a molecule to a change in its electron distributions. All these global reactivity descriptors are required to understand the biological activity of a molecule.

The calculated EA of cytosine is close to the experimental value which extrapolated the electron affinity of the valence anion of bare cytosine to be $0.13 \pm 0.12 \mathrm{eV}[30]$ and the IP is $8.49 \mathrm{eV}$, very close to the experimental value: $8.68 \mathrm{eV}[31]$, indicating that the computational method is feasible. The IP values are similar for zebularine, cytosine and 5-azaHC, except 5-azaC which is less than half of any of the three mentioned. This indicates that 5 -azaC is rather easy to lose an electron. The EA values are rather different and their singly occupied molecular orbitals (SOMO) are shown in Figure 1. The SOMO of zebularine is a $\pi$ orbital with excess electron locating on $\mathrm{C} 5$, corresponding to its reduction potential; more negative than cytosine and 5-azaHC by a few tenths of an eV. The SOMOs of cytosine and 5-azaC are the typical characteristics of the $\mathbb{Z}^{*}$ antibond orbitals. The SOMO of 5-azaHC, the highest in energy, belongs to lone pair electron localized on N5. The negative EA of zebularine and 5-azaC indicate that the anions are electronically unbound at the equilibrium geometries of the corresponding neutral, and they are unstable.

Bisecting the sum of IP and EA values produces the chemical hardness $\eta$. The largest negative EA of zebularine induces the largest $\eta$. The least IP with the EA close to zero of 5-azaC results in the smallest $\eta$. The more hardness of molecules, the more stable they are, as stated, "at equilibrium, chemical systems are as hard as possible" [32]. The $\eta$ value of 5-azaC, half of that of cytosine, is accounting for the fast decomposition in aqueous solution[33,34]. The largest $\eta$ value of zebularine indicates that it is least reactive of the four molecules, whereas the respective $\eta$ values of cytosine and 5-azaHC suggest that they are relatively active. 
Table I. Reactivity parameters concerning ionization potential (IP), electron affinity (EA), and chemical hardness $(\eta)$ of cytosine, 5 -azaC and 5 -azaHC calculated at the M062X/6-311++G(2d,2p) level of theory. Units are in $\mathrm{eV}$.

\begin{tabular}{|llll|}
\hline molecules & IP & EA & $\eta$ \\
\hline cytosine & 8.49 & 0.11 & 4.19 \\
\hline zebularine & 8.85 & -0.33 & 4.59 \\
\hline 5-azaC & 4.10 & -0.08 & 2.09 \\
\hline 5-azaHC & 8.40 & 0.21 & 4.10 \\
\hline
\end{tabular}

The instability of 5-azaC is closely related with $\mathrm{N} 5$ atom. With the electron-lone pair and more electronegativity, more electronic charge accumulates on N5. This makes the angle C4N5C6 a little sharp. It is $113.7^{\circ}$, smaller than the corresponding angle of cytosine $\left(115.9^{\circ}\right)$ and 5 -azaHC $\left(115.0^{\circ}\right)$. The angle of C2N3C4 is $118.7^{\circ}$ for 5 -azaC, the identically moiety in cytosine is $120.4^{\circ}$ and that of 5 -azaHC is $119.0^{\circ}$. The reduced angles increase the triazine tension, causing 5-azaC unstable. In alkaline and water solutions 5-aza-deoxycytidine (Scheme 1) undergoes a rapid and reversible opening of the 5-azacytosine ring, followed by irreversible decomposition[35, 36].

The MESP is a well-established approach for the determination of reactive behaviour of a molecule. The electrostatic potential on individual atoms and the values of three maximum electrostatic potential $\left(V_{\text {max }}\right.$ in orange) and two minimum electrostatic potential $\left(\mathrm{V}_{\mathrm{min}}\right.$, in cyan) of the systems (except zebularine) are presented in Figure 2. The blue surface surrounding some nitrogen and oxygen atoms represents the region of maximum electro negativity and the red colour surface accumulated on some hydrogen atoms shows their electro positivity. The $\mathrm{V}_{\min }$ values vary from -48.6 to $-54.5 \mathrm{kcal} / \mathrm{mol}$ and large or small $\mathrm{V}_{\max }$ values scatter around $\mathrm{N}-\mathrm{H}$ or $\mathrm{C}-\mathrm{H}$ hydrogens. If we compare the MESP of zebularine and cytosine, we can observe that both the three $\mathrm{V}_{\max }$ and one $\mathrm{V}_{\min }$ are slightly smaller for zebularine (37.8, 27.5, 20.7 and $-49.8 \mathrm{kcal} / \mathrm{mol}$, respectively) than for cytosine $(48.4,36.8,33.8$ and $-53.5 \mathrm{kcal} / \mathrm{mol}$, respectively). The lesser basicity of N3 atom makes zebularine unfavourable to be protonated and unsusceptible to enzyme-mediated reaction[37, 38]. Second, the electrostatic potential around C5 in cytosine is -5.0 $\mathrm{kcal} / \mathrm{mol}$, while it is $-0.03 \mathrm{kcal} / \mathrm{mol}$ for zebularine, the less nucleophilicity impedes DNA methyltransferases enzymes to methylate the $\mathrm{C} 5$ position when zebularine acts as a substrate. Lastly, the absence of the hydrogen bond interactions formed by the exocyclic amino group makes zebularine difficult to locate in a right position for the nucleophilic addition[8]. Compared to zebularine, the amino $\mathrm{N}$ atom enhances $\pi$ electron delocalization around cytosine, producing more positive $V_{\max }$ and more negative $V_{\min }$ and the consequent favourable substrate for methylation. The amino hydrogen atoms are 
likely to transfer to N3 atom for its strong proton affinity, forming tautomers of E/Z conformations (Cyt-E/ Cyt-Z, see SI). Tautomerization of cytosine is less abundant, since the amino hydrogens possess relatively low $\mathrm{V}_{\max }$ (compared to those of 5 -azaHC). The Boltzmann population of the Cyt-E and Cyt-Z tautomers is $5.9 \%$, close to the experiment that traces of the imino population ( 12\%) exist in 1-methylcytosine vapor[33, 34]. The Cyt-Z conformation is relatively more populated than Cyt-E, for the easier abstraction of the amino hydrogen with a more positive MESP potential of $48.4 \mathrm{kcal} / \mathrm{mol}$ around.

For 5 -azaC, the replacement of the $\mathrm{C}-\mathrm{H}$ group by a nitrogen atom provides an additional basic center $(-20.0 \mathrm{kcal} / \mathrm{mol})$ which in turn affects the basicity of the other nitrogen atoms of the ring (Figure 1c). The MESP distribution around 5-azaC, whether $\mathrm{V}_{\max }$ or $\mathrm{V}_{\min }$, are both lower than those in cytosine. The second $V_{\text {min }}$ of cytosine lies on the amino $\mathrm{N}$ atom, whereas 5 -azaC provides the second $\mathrm{V}_{\text {min }}$ near $\mathrm{N} 5$ atom. At this site, methylation can be formed by a covalent bond; however, $\beta$-elimination of DNMT1 (DNA (cytosine-5)-methyltransferase 1) cannot occur due to the lack of a hydrogen atom at this position[39, 40]. Consequently, methylation of DNMT1 is unable to continue. In the case of 5-azaHC, the MESP is redistributed upon the saturation of $\mathrm{N} 5$ and $\mathrm{C} 6 . \pi$ electrons are more localized within $\mathrm{N} 3$ and the oxygen atom, leading to gained $V_{\max }$ and $V_{\min }$ values (Figure $1 \mathrm{~d}$ ). The sites as well as their $\mathrm{V}_{\max }$ values (53.9, $52.1 \mathrm{kcal} / \mathrm{mol}$ ) of the amide and amino hydrogens are very close. This intramolecular proton-proton repulsion causes increased proton kinetic energy, which would enhance isomerization rates. Seven tautomers were observed in the experiment[41], and their respective structures are listed in the SI.

\section{Conclusion}

In summary, $\mathrm{C} 5$ is a docking site for methylation for cytosine. Nitrogen substitution at this site makes zebularine and 5-azaC an inhibitor for methyl addition, while 5-azaHC has multiple tautomers for the active hydrogen atom on $\mathrm{N} 5$ and this molecule acts as a mutagenesis treatment. Herein, is can be found that the effects of chemical modifications of cytosine impinge great influences on biological activities. Chemical modification of natural nucleobases opens a new field in the search for effective antiviral and antitumor therapy. In this work, MESP and molecular hardness are valuable tools for description of molecular activities and can give reasonable information for the experiments.

\section{Declarations}

Funding: (Funding from Heze University)

Conflicts of interest/Competing interests: (No)

Availability of data and material: (All data generated or analysed during this study are included in this published article and its supplementary information files)

Code availability: (No)

Authors' contributions: (Guixiu Wang carried out the computational work. Yifang Wu drew all pictures.) 


\section{References}

1. Co EW, Henschke JP (2016) The Development and Manufacture of Azacitidine, Decitabine, and Cladribine: Stereoselective Ribonucleoside Drug Synthesis Using the Vorbrüggen Glycosylation. In: Comprehensive Accounts of Pharmaceutical Research and Development: From Discovery to LateStage Process Development Volume, 2. American Chemical Society, pp 271-321

2. Brookes P, Lawley PD (1962) 254. The methylation of cystosine and cytidine. J Chem Soc 13481351

3. Seley-Radtke KL, Yates MK (2018) The evolution of nucleoside analogue antivirals: A review for chemists and non-chemists. Part 1: Early structural modifications to the nucleoside scaffold. Antiviral Res 154:66-86

4. Smith SS, Kaplan BE, Sowers LC, Newman EM (1992) Mechanism of human methyl-directed DNA methyltransferase and the fidelity of cytosine methylation. Proc Natl Acad Sci U S A 89:4744-4748

5. Chen L,., Macmillan AM, Chang W, ., Ezaz-Nikpay K, ., Lane WS, Verdine GL (1991) Direct identification of the active-site nucleophile in a DNA (cytosine-5)-methyltransferase. Biochemistry 30:11018

6. Reinisch KM, Chen L, Verdine GL, Lipscomb WN (1995) The crystal structure of Haelll methyltransferase covalently complexed to DNA: An extrahelical cytosine and rearranged base pairing. Cell 82:143-153

7. Maslov AY, Lee M, Gundry M, Gravina S, Strogonova N, Tazearslan C, Bendebury A, Suh Y, Vijg J (2012) 5-Aza-2'-deoxycytidine-induced genome rearrangements are mediated by DNMT1. Oncogene $31: 5172-5179$

8. Aranda J, Attana F, Tuñón I (2017) Molecular Mechanism of Inhibition of DNA Methylation by Zebularine. ACS Catal 7:1728-1732

9. Bowler EH, Bell J, Divecha N, Skipp P, Ewing RM (2019) Proteomic Analysis of Azacitidine-Induced Degradation Profiles Identifies Multiple Chromatin and Epigenetic Regulators Including Uhrf1 and Dnmt1 as Sensitive to Azacitidine. J Proteome Res 18:1032-1042

10. Chang HW, Hui-Chun W, Chiau-Yi C, Ting-Wei H, Ming-Feng H, Shyng-Shiou Y, Chih-Jen H, Chao-Neng T (2014) 5-Azacytidine Induces Anoikis, Inhibits Mammosphere Formation and Reduces Metalloproteinase 9 Activity in MCF-7 Human Breast Cancer Cells. Molecules 19:3149-3159

11. Gryn J, Zeigler ZR, Shadduck RK, Lister J, Raymond JM, Sbeitan I, Srodes C, Meisner D, Evans C (2002) Treatment of myelodysplastic syndromes with 5-azacytidine. Leuk Res 26:893-897

12. Raj K, Mufti GJ (2007) Azacytidine (Vidaza(R)) in the treatment of myelodysplastic syndromes. Ther Clin Risk Manag 2:377-388

13. Futami K, Maita M, Katagiri T (2019) DNA demethylation with 5-aza-2'-deoxycytidine induces the senescence-associated secretory phenotype in the immortal fish cell line. EPC Gene 697:194-200

14. Loeb LA, Essigmann JM, Kazazi F,., Zhang J, Rose, Mullins KD JI (1999) Lethal mutagenesis of HIV with mutagenic nucleoside analogs. Proc Natl Acad Sci USA 96:1492-1497 
15. Harris KS, Brabant W, Styrchak S, Gall A, Daifuku R (2005) KP-1212/1461, a nucleoside designed for the treatment of HIV by viral mutagenesis. Antiviral Res 67:1-9

16. Eigen M (2002) Error catastrophe and antiviral strategy. Proc Natl Acad Sci U S A 99:13374-13376

17. Hohenberg P, Kohn W (1964) Inhommogeneous Electron Gas. Phys Rev 136:864-871

18. Chandrakumar KRS, Ghanty TK, Ghosh SK (2004) Relationship between Ionization Potential, Polarizability, and Softness: A Case Study of Lithium and Sodium Metal Clusters. J Phys Chem A 108:6661-6666

19. Parr RG, Yang W (1989) Density Functional Theory of Atoms and Molecules. Oxford University Press, New York

20. Murray JS, Clark T, Riley KE, Politzer P (2012) $\sigma$-Holes, $\pi$-holes and electrostatically-driven interactions. J Mol Model 18:541-548

21. Politzer P, Murray J (2021) Electrostatic potentials at the nuclei of atoms and molecules. Theor Chem Acc. 140

22. Bijina PV, Suresh CH (2020) Molecular Electrostatic Potential Reorganization Theory to Describe Positive Cooperativity in Noncovalent Trimer Complexes. J Phys Chem A 124:2231-2241

23. Mohan N, Suresh CH (2014) Anion Receptors Based on Highly Fluorinated Aromatic Scaffolds. J Phys Chem A 118:4315-4324

24. Dimitrova V, llieva S, Galabov B (2003) Electrostatic potential at nuclei as a reactivity index in hydrogen bond formation. Complexes of ammonia with $\mathrm{C}-\mathrm{H}, \mathrm{N}-\mathrm{H}$ and $\mathrm{O}-\mathrm{H}$ proton donor molecules. J Mol Struct: Theochem 637:73-80

25. Dimitrova M, llieva S, Galabov B (2003) Reactivity descriptors for the hydrogen bonding ability of aliphatic alcohols. J Mol Struct 657:317-324

26. Jia X, Li P (2019) Solvation Free Energy Calculation Using a Fixed-Charge Model: Implicit and Explicit Treatments of the Polarization Effect. J Phys Chem B 123:1139-1148

27. Huff AK, Mackenzie RB, Smith CJ, Leopold KR (2019) A Perfluorinated Carboxylic Sulfuric Anhydride: Microwave and Computational Studies of CF3COOSO2OH. J Phys Chem A 123:2237-2243

28. 09 G In: M. J. Frisch GWT, H. B. Schlegel, G. E. Scuseria„ M. A. Robb JRC, G. Scalmani, V. Barone, B. Mennucci,, G. A. Petersson HN, M. Caricato, X. Li, H. P. Hratchian,, A. F. Izmaylov JB, G. Zheng, J. L. Sonnenberg, M. Hada, M. Ehara KT, R. Fukuda, J. Hasegawa, M. Ishida, T. Nakajima, Y. Honda OK, H. Nakai, T. Vreven, J. A. Montgomery, Jr.,, et al., editors. Revision E.01 ed. Wallingford CT: Gaussian, Inc; 2013

29. Pearson RG (1985) Absolute electronegativity and absolute hardness of Lewis acids and bases. J Am Chem Soc 107:6801-6806

30. Schiedt J, Weinkauf R, Neumark DM, Schlag EW (1998) Anion spectroscopy of uracil, thymine and the amino-oxo and amino-hydroxy tautomers of cytosine and their water clusters. Chem Phys 239:511-524 
31. Orlov VM, Smirnov AN, Varshavsky YM (1976) Ionization potentials and electron-donor ability of nucleic acid babes and their analogues. Tet Lett 17:4377-4378

32. Parr RG, Chattaraj PK (1991) Principle of Maximum Hardness. J Am Chem Soc 113:1854-1855

33. Szczesniak M, Leszczynski J, Person WB (1992) Identification of the imino-oxo form of 1methylcytosine. J Am Chem Soc 114:2731-2733

34. Smets J, Adamowicz L, Maes G (1996) Matrix-Isolation FT-IR Studies and ab Initio Calculations of Hydrogen-Bonded Complexes of Molecules Modeling Cytosine or Isocytosine Tautomers. 5. 1-CH3Cytosine Complexes with H2O in Ar Matrices. J Phys Chem 100:6434-6444

35. Lin KT, Momparlerm RL, Rivard GE (1981) High-performance Liquid Chromatographic Analysis of Chemical Stability of 5-aza-2'-Deoxycytidine. J Pharm Sci 70:1228-1232

36. Rogstad DK, HJ L, Theruvathu JA, Theruvathu AB (2009) Chemical decomposition of 5-aza-2'deoxycytidine (Decitabine): kinetic analyses and identification of products by NMR, HPLC, and mass spectrometry. Chem Res Toxicol 22:1194-1204

37. Peräkylä M (1998) A Model Study of the Enzyme-Catalyzed Cytosine Methylation Using ab Initio Quantum Mechanical and Density Functional Theory Calculations: pKa of the Cytosine N3 in the Intermediates and Transition States of the Reaction. J Am Chem Soc 120:12895-12902

38. Aranda J, Zinovjev K, Świderek K, Roca M, Tuñón I (2016) Unraveling the Reaction Mechanism of Enzymatic C5-Cytosine Methylation of DNA. A Combined Molecular Dynamics and QM/MM Study of Wild Type and GIn119 Variant. ACS Catal 6:3262-3276

39. Stresemann C, F. L (2008) Modes of action of the DNA methyltransferase inhibitors azacytidine and decitabine[J]. International journal of cancer. Journal international du cancer, 2008, 123(1):8-13. Int J Cancer.123:8-13

40. Shelton J, Lu X, Hollenbaugh JA, Cho JH, Amblard F, Schinazi RF (2016) Metabolism, Biochemical Actions, and Chemical Synthesis of Anticancer Nucleosides, Nucleotides, and Base Analogs. Chem Rev 116:14379-14455

41. Deyu L, Fedeles BI, Vipender S, Chunte Sam P, Silvestre KJ, Simi AK, Simpson JH, Andrei T, Essigmann JM (2014) Tautomerism provides a molecular explanation for the mutagenic properties of the anti-HIV nucleoside 5-aza-5,6-dihydro-2'-deoxycytidine. Proc Natl Acad Sci USA 111:32523259

\section{Figures}



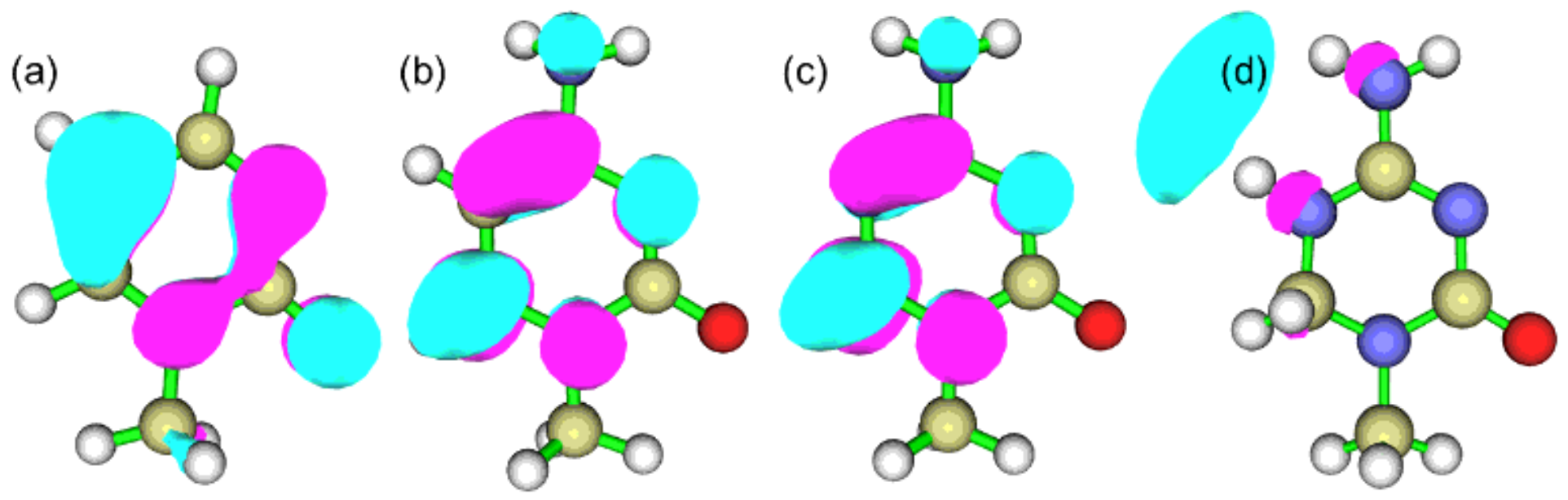

\section{Figure 1}

The singly occupied molecular orbital (SOMO) of (a) zebularine, (b) cytosine, (c) 5-azaC and (d) 5-azaHC. The isovalue is 0.05 Bohr for (a), (b) and (c). It is 0.04 Bohr for (d). 

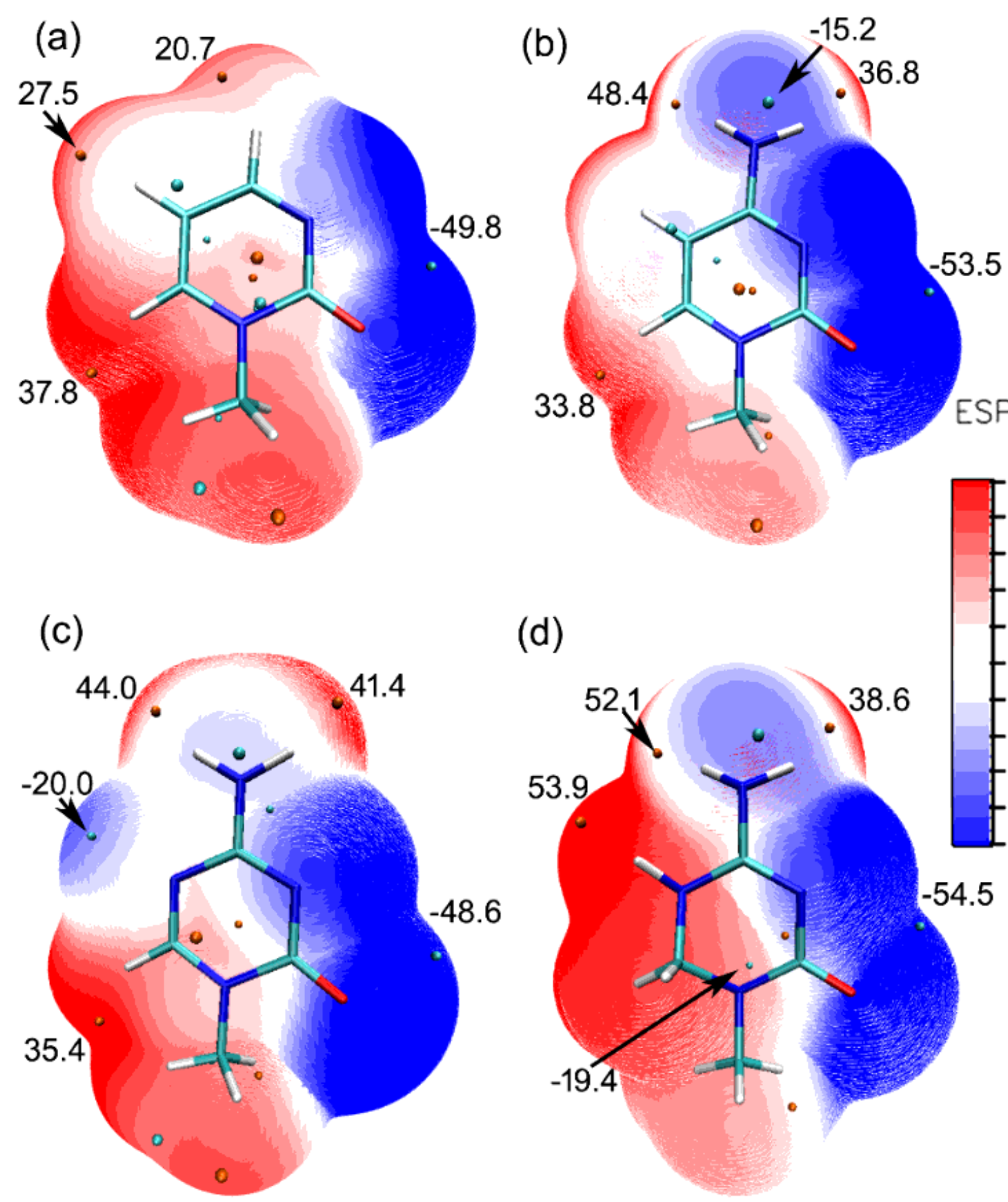

\section{Figure 2}

Molecular electrostatic potential isosurfaces for (a) zebularine, (b) cytosine, (c) 5-aza-C, and 5-aza-2HC. The electrostatic potential dots are coloured orange (positive) and cyan (negative), and the corresponding Vmax and Vmin values are given in kcal mol-1.

\section{Supplementary Files}


This is a list of supplementary files associated with this preprint. Click to download.

- Scheme1.png 